\title{
The role and significance of sensitivity analyses in enhancing the statistical validity of clinical studies
}

Michael Baiocchi, PhD, ${ }^{\mathrm{a}}$ Y. Joseph Woo, MD, ${ }^{\mathrm{b}}$ Peter Chiu, MD, ${ }^{\mathrm{b}}$ and Andrew B. Goldstone, MD, PhD ${ }^{\mathrm{b}}$

As surgeons, we operate with the intent of causing a patient's health to improve. In statistical jargon, we are interested in the causal effect of one treatment in contrast to another, for example, for a mitral valve in need of replacement, will a patient survive longer with a mechanical or bioprosthetic valve? ${ }^{1}$ The surest way to generate useful data to answer a causal question is to run a randomized controlled trial (RCT). There are many nuances, but it is largely true that RCTs produce strong causal conclusions because the necessary assumptions are true by design; that is, the statistical assumptions needed to obtain reliable causal estimates are made true by researchers randomizing people into treatment $\mathrm{A}$ or treatment $\mathrm{B}$. When we cannot conduct an RCT, we must generate causal insights from observational data. In causal observational studies, where the data were not generated through intentional randomization, we invoke assumptions to estimate causal effects. If these assumptions are not guaranteed to be true the way they are in an RCT, then what happens when we know a particular assumption is assailable? It turns out that a violation of an assumption is not equally destructive to all observational studies. Some observational studies rely heavily on an assumption, whereas other studies can withstand substantial deviations from that assumption. We can quantify this resilience to bias using sensitivity analyses.

The goal of this article is to supply the reader with strong intuitions about sensitivity analyses-what they do, what they do not do (but we often wish they could), and how to interpret them. We assume the reader is familiar with ideas of bias, confounding, and propensity score methods. We also assume this is the first time readers are being introduced to modern, formal sensitivity analyses. Sometimes one will see ad hoc analyses called "sensitivity analyses" in articles, for example, comparing a logistic regression with 10 variables to

\footnotetext{
From the ${ }^{\mathrm{a}}$ Department of Epidemiology and Population Health, Stanford University, Stanford, Calif; and ${ }^{\mathrm{b}}$ Department of Cardiothoracic Surgery, Stanford University, Stanford, Calif.

Received for publication July 6, 2020; revisions received Aug 31, 2020; accepted for publication Sept 3, 2020; available ahead of print Oct 19, 2020.

Address for reprints: Michael Baiocchi, PhD, Redwood Building, T253, 150 Governor's Lane, Stanford, CA 94305-5405 (E-mail: baiocchi@stanford.edu).

J Thorac Cardiovasc Surg 2022;163:749-53

$0022-5223 / \$ 36.00$

Copyright (C) 2020 by The American Association for Thoracic Surgery

https://doi.org/10.1016/j.jtcvs.2020.09.134
}

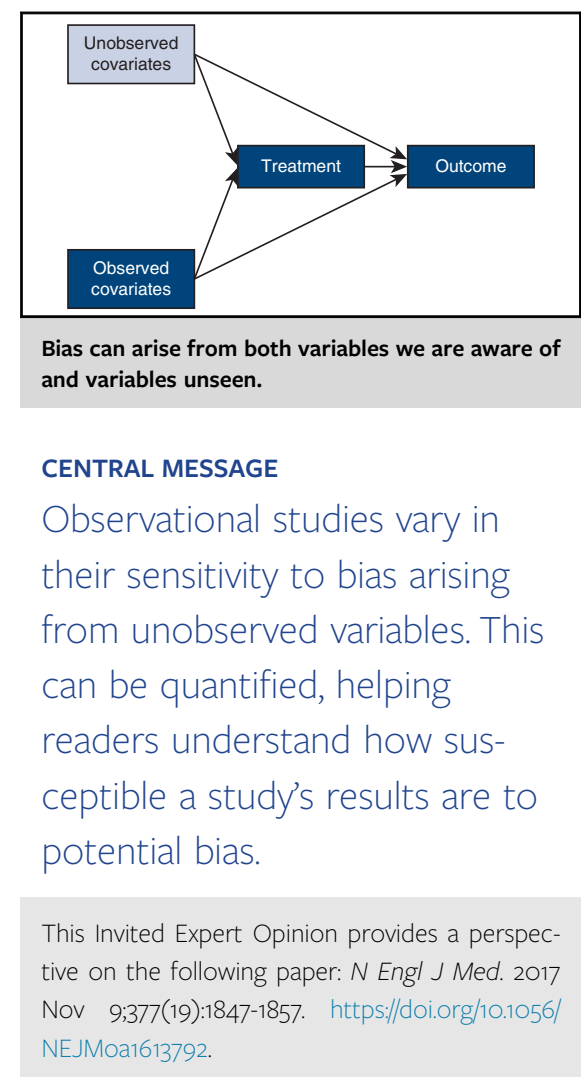

See Commentaries on pages 754 and 755 .

one with 15 or re-running an analysis after removing "problematic" observations; these are not sensitivity analyses in the modern sense. Formal sensitivity analyses quantify the impact of violating a particular assumption. This article will not prove to the reader that these formal sensitivity analyses are valid (references exist ${ }^{2-5}$ ). An important takeaway from this article is that there is not an all-or-nothing brittleness to observational studies; we can provide, and should provide, quantitative measurements of how sensitive an observational study is to bias so readers can judge how easily the conclusions could be changed because of a violation of an assumption.

\section{PROPENSITY SCORE ASSUMPTIONS}

We assume readers are familiar with propensity score methods and refer readers interested in more details to 2 
clinical primers: one in the Journal ${ }^{6}$ that focuses on matching methods and another ${ }^{7}$ that additionally discusses weighting using the propensity score.

Strongly ignorable treatment assignment (SITA) is the assumption that the observed pretreatment variables (eg, age and comorbidities of patient) in Figure 1 are sufficient to describe all of the selections into a treatment type, that is, there are no unobserved variables as shown in Figure 1 (eg, frailty). This assumption is often hard to defend. In fact, clinicians work hard against the SITA assumption: Clinicians use all available information about a patient's health to select the best treatment for that patient. Clinicians' judgments are about as far away from a randomization as possible, much to the patient's relief and the analyst's frustration. SITA is the assumption we interrogate using a sensitivity analysis in the next section.

Propensity score methods work because, assuming SITA, they give us a way of designing our study such that the data behave as if they came from an RCT. Propensity scores focus on understanding how patients were sorted into treatment, which is then used to sever the pretreatment variables' ability to falsely create an impression that there is a causal effect. By matching, weighting, or stratifying with the propensity score, and given the necessary assumptions are true, we can use traditional methods of inference (eg, regression, Fisher exact tests) to estimate causal effects from observational data. The clever bit about sensitivity of SITA is that statisticians have figured out a way to quantify the required "departure from as-if random treatment assignment" before a study's conclusions are dubious. As argued by the cocreator of propensity scores, ${ }^{2}$ an analysis that relies on SITA is incomplete without a sensitivity analysis.

\section{Sensitivity Analyses}

There are several approaches for doing sensitivity analyses of the SITA assumption when using propensity scores. For weighting-based designs, there is the alpha sensitivity. ${ }^{3}$ For matching designs, there is gamma sensitivity. ${ }^{2}$ More recently, a generalized framework for sensitivity analysis has been proposed ${ }^{4}$; the E-value approach to sensitivity

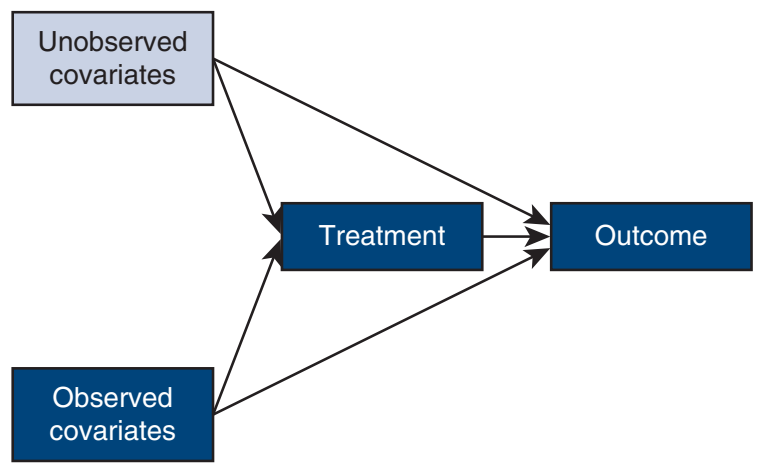

FIGURE 1. A directed acyclic graph, where arrows indicate the impact of one variable on another. analysis appears to be quite flexible and may catch on more broadly in clinical and health services research. Given the prevalence of propensity score matching in the thoracic and cardiovascular surgery literature, and the depth of development of gamma sensitivity in the statistics community, we focus on the sensitivity analysis for studies that use matching. Beyond challenges to the internal validity of a study (of which SITA violations are one), there has also been useful work ${ }^{8,9}$ understanding and quantifying challenges to external validity (aka "generalizability"), particularly the Tipton index. ${ }^{10}$

In propensity score matching, we create matched sets of people who have the same propensity score, but within the set, some have treatment A and some have treatment B. In practice, we do this matching without any guarantee that the SITA assumption holds. For example, say we conducted a study and used 40 observed covariates (which is a lot) to create our propensity score, but we did not have a variable (or variables) particularly relevant to the sorting of patients into a treatment type. If this violation of SITA is true, then even though we matched using propensity scores, within each matched set the patients no longer behave as if they were randomized to treatment A or B. Instead, there is often some residual preferential selection of "sicker" patients into one of the treatment types. Instead of a "fair" randomization (each person within the set equally likely to get treatment A), we now have a randomization that pushes sicker patients within a set into one of the treatments at a higher rate. This is the key insight of a gamma sensitivity analysis: We can quantify how sensitive a study is to unobserved variables by quantifying how much of a departure from a fair randomization it would take before the study's conclusions are no longer qualitatively defensible.

The departure from as-if random is described by a parameter called the "gamma sensitivity" of a study. The parameter, usually just referred to as "gamma," goes from 1 to infinity. A gamma $=1$ is meant to describe a study where the data behave as if they came from an RCT. A gamma $=1.1$ is a study where the data have a bit of a departure from an RCT. A gamma $=4$ is a scenario where there is extremely serious preferential selection of the "sick patients" into one of the treatment types. In practice, once we have completed our propensity score analysis we can then calculate the gamma sensitivity of the study and assert something such as "This particular study's conclusion is insensitive to bias arising from unobserved variables up to a gamma $=1.4$." We will talk more about exactly how to interpret the gamma in the next section, but the first takeaway is already clear: The larger gamma that the study can withstand, the more robust the study's conclusions are to unobserved variables causing bias.

For any given data set, there is a maximum achievable gamma. Yet we are not guaranteed to achieve that maximal gamma. In fact, given the same data set and task, 2 analysts 
are likely to achieve different gammas through designing their studies differently. There are ways to design an observational study making it more or less resistant to challenges arising from unobserved variables. The basic principles of designing a study that achieves a higher gamma are not terribly tricky; we refer interested readers to 3 accessible articles to learn how to design robust studies. ${ }^{11-13}$

There are several convenient statistical packages for performing these analyses in $\mathrm{R}$ (eg, sensitivitymw ${ }^{14}$ and Rbounds $^{15}$ ), SAS (eg, PSMATCH), and STATA (eg, RBOUNDS ${ }^{16}$ ). Information on other methods and software is available in Table $1 .^{1-4,11,17-19,20,22,23}$ These references contain sample code for implementing.

\section{Interpreting a Gamma Sensitivity Analysis}

One way to interpret the gamma sensitivity of a study is for 2 people who look alike in our data set (eg, have the same propensity score), the gamma sensitivity of a study is the odds ratio of the actual probability of assignment to treatment A that it would take to change the qualitative conclusions of the study. Gamma is tracking the departure from what our observed variables seem to imply and the actual probability of receiving a treatment. For example, say we think person 1 and person 5 both have a propensity score of 0.25 . We match them together. Unbeknownst to us, there were unobserved variables we did not account for, and the true probability of assignment to treatment for person 1 is 0.30 and person 5 is 0.25 ; then their actual odds ratio is $(0.30 /(1-0.30)) /(0.25 /(1-0.25))=1.29$. If our study had a gamma sensitivity of greater than 1.29 , then this kind of mistake in our matching would not be problematic to the study's conclusion. This is not the most natural way for people or reviewers to think; it is easier to point out the absence of a variable that should have been accounted for. Yet now we can ask a more sophisticated question: How damaging is this absence to the overall conclusions of the study? If we can quantify this, then the critic will need to work a bit harder to make a claim that this variable's (or these variables') absence could plausibly call into question our study's conclusion.

Unfortunately, unlike for $P$ values where there is agreement on cutoff points that have meaning (eg, $P<.05$ ), there is not currently a consensus about what constitutes a cutoff point for a gamma. It would be nice if the literature had a "strong enough" cutoff point for gamma sensitivities.

It is hard to set a one-size-fits-all cutoff for a gamma sensitivity parameter because of how context specific it is. For a particular study, if the analyst can achieve a larger gamma then that is preferred to a smaller one because it means that the study is less sensitive to having its conclusions manipulated by unobserved variables. Comparing the gammas from 2 different observational studies is difficult. Take 2 studies that compare treatments A and B but include different variables in their propensity score model. If one study includes 40 variables that capture all pretreatment clinical variables that physicians consider when assigning treatment, then only variables we are unaware of-and are uncorrelated with the variables we do haveremain of concern us (eg, economic factors, communication issues, unconscious tendencies). In this setting, critics will have fewer well-articulated challenges that must be fended off by the gamma, so a smaller gamma is less alarming. Alternatively, if critics look at the list of just 5 pretreatment variables included in the second study and finds it lacking, then they will be able to articulate several unobserved variables that might have caused sorting between treatments. A very large gamma will be required to defend that study's conclusions.

\section{DISCUSSION}

We offer 3 summarizing statements about sensitivity analyses: (1) what sensitivity analyses tell you, (2) what they do not tell you (but we wish they would), and (3) an analogy that may be helpful for understanding this difference. A sensitivity analysis tells you how much of a violation of an assumption a particular study can withstand before the

TABLE 1. References for common sensitivity analyses

\begin{tabular}{|c|c|c|c|c|}
\hline Name & Use & Reference & Surgery example & Software \\
\hline Gamma & $\begin{array}{l}\text { Propensity score matching } \\
\text { 1-time treatment }\end{array}$ & $\begin{array}{l}\text { Rosenbaum }^{2} \\
\text { Chapters } 3 \text { and } 14\end{array}$ & Goldstone and colleagues $^{21}$ & $\begin{array}{l}\text { R: sensitivitymw }{ }^{11} \\
\text { SAS: PSMATCH }\end{array}$ \\
\hline Alpha & $\begin{array}{l}\text { Propensity score weighting } \\
\text { 1-time treatment or repeated } \\
\text { treatments }\end{array}$ & Brumback and colleagues $^{3}$ & Goldstone and colleagues ${ }^{1}$ & $\begin{array}{l}\text { R: Causalsens }{ }^{18} \\
\text { SAS }^{*}\end{array}$ \\
\hline E-value & $\begin{array}{l}\text { Useful for most models of 1-time } \\
\text { treatment effects }\end{array}$ & VanderWeele and Ding ${ }^{4}$ & Anderson and colleagues ${ }^{22}$ & $\begin{array}{l}\text { R: E-value }{ }^{19} \\
\text { STATA: E-value }{ }^{20}\end{array}$ \\
\hline Greenland & $\begin{array}{l}\text { Noncausal sensitivity analysis } \\
\text { useful for correlations of binary } \\
\text { outcome with binary treatment }\end{array}$ & Greenland $^{17}$ & Eliasson and colleagues $^{23}$ & $\begin{array}{l}\text { Not available, but simple to } \\
\text { calculate }\end{array}$ \\
\hline
\end{tabular}

*There does not appear to be a formal package for implementing alpha sensitivity in SAS, but sample code implementing a simplified version of alpha does appear to be available from one source. ${ }^{1}$ 
study's conclusion is called into doubt. This is scientifically valuable because it shifts the debate away from an all-ornothing brittleness (eg, "You didn't control for this variable therefore this study cannot be trusted") to a more useful debate about how variables influence each other (ie, we acknowledge that flaws can exist without completely undermining the overall conclusions of the analysis). Unfortunately, a sensitivity analysis does not estimate the amount of a violation that has occurred, but this would be a nice quantity to have. It would be useful to be able to make a statement such as "Using the data we have, we can see that there are $\mathrm{X}$ number of variables omitted and they have an impact of Y on our estimation of the causal effect." That kind of statement is not obtainable in general. We cannot usually know the impact of the variables we cannot observe. Instead, a sensitivity analysis reports how insensitive the study's conclusions are, were there to be hypothetical violations. An analogy for these contrasting points is to consider a bridge. An engineer looks at the materials it is constructed out of and the design of the bridge. The engineer then estimates how large an earthquake the bridge could withstand, perhaps a magnitude 7.5 on the Richter scale. We cannot look at the bridge and understand what kind of earthquake will happen. We would need to collect different kinds of data and perform different kinds of analyses to get that information. Instead, presented with the engineer's estimate, it is our job to assess the context of the bridge and debate whether its sturdiness is sufficient. Perhaps this would be acceptable for a bridge spanning a peaceful river in a tectonically settled area, but in the San Francisco Bay Area one might need to think carefully before proceeding to place one's trust in this bridge.

This article focused on propensity score-based methods and the sensitivity analyses that evaluate violations of the SITA assumption. There are other study designs, such as instrumental variables, that do not use the SITA assumption but rather address unobserved variables directly through a form of naturally occurring randomization. Interested readers can read more about instrumental variables in a surgical context from this Journal ${ }^{24}$ or more broadly in health services research. ${ }^{25}$ Readers of this Journal who are interested in how propensity score methods contrast with instrumental variable approaches might explore some work our team did on understanding the impact of regionalizing care of acute type A aortic dissection. ${ }^{21}$ In that study, the primary analysis makes use of an instrumental variable design, but in the supplement we discuss the results of reanalyzing the data using a propensity score design and how these 2 designs differ in their ability to address both observed and unobserved variables. Further, in that article, there are several uses of sensitivity analysis: for instrumental variables, propensity scores, and censored observations.

\section{CONCLUSIONS}

The majority of surgical research is observational. Cardiothoracic surgeons appropriately adopted sophisticated techniques, such as propensity score matching and weighting, to improve estimates of causal effects. However, if we want a robust literature that uses observational data in responsible ways, then we need to understand the assumptions we make and use formal sensitivity analyses to quantify resilience to violations of these assumptions.

\section{Conflict of Interest Statement}

The authors reported no conflicts of interest.

The Journal policy requires editors and reviewers to disclose conflicts of interest and to decline handling or reviewing manuscripts for which they may have a conflict of interest. The editors and reviewers of this article have no conflicts of interest.

\section{References}

1. Goldstone AB, Chiu P, Baiocchi M, Lingala B, Patrick WL, Fischbein MP, et al. Mechanical or biologic prostheses for aortic-valve and mitral-valve replacement. N Engl J Med. 2017;377:1847-57.

2. Rosenbaum PR. Design of Observational Studies. New York: Springer; 2010.

3. Brumback BA, Hernán MA, Haneuse SJ, Robins JM. Sensitivity analyses for unmeasured confounding assuming a marginal structural model for repeated measures. Stat Med. 2004;23:749-67.

4. VanderWeele TJ, Ding P. Sensitivity analysis in observational research: introducing the E-value. Ann Intern Med. 2017;167:268-74.

5. Liu W, Kuramoto SJ, Stuart EA. An introduction to sensitivity analysis for unobserved confounding in nonexperimental prevention research. Prev Sci. 2013;14: 570-80.

6. McMurry TL, Hu Y, Blackstone EH, Kozower BD. Propensity scores: methods, considerations, and applications in the Journal of Thoracic and Cardiovascular Surgery. J Thorac Cardiovasc Surg. 2015;150:14-9.

7. Brookhart MA, Wyss R, Layton JB, Stürmer T. Propensity score methods for confounding control in nonexperimental research. Circ Cardiovasc Qual Outcomes. 2013;6:604-11.

8. Westreich D, Edwards JK, Lesko CR, Stuart E, Cole SR. Transportability of trial results using inverse odds of sampling weights. Am J Epidemiol. 2017;186: 1010-4.

9. Cole SR, Stuart EA. Generalizing evidence from randomized clinical trials to target populations: the ACTG 320 trial. Am J Epidemiol. 2010;172:107-15.

10. Tipton E. How generalizable is your experiment? An index for comparing experimental samples and populations. J Educ Behav Stat. 2014;39:478-501.

11. Rosenbaum PR. Heterogeneity and causality: unit heterogeneity and design sensitivity in observational studies. Am Stat. 2005;59:147-52.

12. Leacy FP, Stuart EA. On the joint use of propensity and prognostic scores in estimation of the average treatment effect on the treated: a simulation study. Stat Med. 2014;33:3488-508

13. Aikens RC, Greaves D, Baiocchi M. A pilot design for observational studies: using abundant data thoughtfully. Stat Med. 2020 [In press].

14. Rosenbaum PR. Two R packages for sensitivity analysis in observational studies. Observational Stud. 2015;1:1-7.

15. Keele L. An Overview of rbounds: An R Package for Rosenbaum Bounds Sensitivity Analysis With Matched Data. White Paper. Columbus, OH; 2010.

16. Gangl M. RBOUNDS: Stata module to perform Rosenbaum sensitivity analysis for average treatment effects on the treated. Boston, MA: Boston College Department of Economics; 2004.

17. Greenland S. Basic methods for sensitivity analysis of biases. Int J Epidemiol. 1996;25:1107-16.

18. Blackwell M. A selection bias approach to sensitivity analysis for causal effects. Polit Anal. 2014;22:169-82. 
19. Linden A, Mathur MB, VanderWeele TJ. Conducting sensitivity analysis for unmeasured confounding in observational studies using E-values: the evalue package. Stata J. 2020;20:162-75.

20. Faries D, Zhang X, Kadziola Z, Siebert U, Kuehne F, Obenchain RL, et al. Real World Health Care Data Analysis: Causal Methods and Implementation Using SAS. Cary, NC: SAS Institute Inc; 2020.

21. Goldstone AB, Chiu P, Baiocchi M, Lingala B, Lee J, Rigdon J, et al. Interfacility transfer of Medicare beneficiaries with acute type A aortic dissection and regionalization of care in the United States. Circulation. 2019;140:1239-50.

22. Anderson MR, Tabah A, RoyChoudhury A, Lederer DJ. Procedure preference and intention-to-treat outcomes after listing for lung transplantation among US adults. A cohort study. Ann Am Thorac Soc. 2019;16:231-9.
23. Eliasson B, Liakopoulos V, Franzén S, Näslund I, Svensson AM, Ottosson J, et al Cardiovascular disease and mortality in patients with type 2 diabetes after bariatric surgery in Sweden: a nationwide, matched, observational cohort study. Lancet Diabetes Endocrinol. 2015;3:847-54.

24. Bagiella E, Karamlou T, Chang H, Spivack J. Instrumental variable methods in clinical research. J Thorac Cardiovasc Surg. 2015;150:779-82.

25. Baiocchi M, Cheng J, Small DS. Instrumental variable methods for causal inference. Stat Med. 2014;33:2297-340.

Key Words: bias, causality, confounding, matching, propensity scores 